\title{
INFORMES*
}

\section{La seguridad de los productos de consumo en Andalucía. (Decreto 5/2009, de 13 de Enero) ${ }^{1}$}

I.- El Decreto 5/2009, de 13 de enero (BOJA núm. 20, de 30 de enero), crea la Red de Alerta de Andalucía de Productos de Consumo y regula la adopción de medidas administrativas preventivas. Desarrolla, por una parte, la Ley 13/2003, de 17 de diciembre, de Defensa y Protección de los Consumidores y Usuarios de Andalucía en lo que concierne a las medidas administrativas preventivas y a las advertencias y requerimientos. Y, por otra, desarrolla la legislación estatal en tales materias, contenida en el Real Decreto 1801/2003, de 26 de diciembre, sobre seguridad general de los productos, dictado con carácter básico (disposición final primera) ${ }^{2}$ y que transpone la Directiva 2001/95/CE, del Parlamento y del Consejo, de 3 de diciembre de 2001, relativa a la seguridad general de los productos.

Como se desprende de su rúbrica, son dos fundamentalmente las materias que regula: la Red de Alerta de Andalucía y las medidas preventivas ante situaciones de riesgo inaceptable para la seguridad de las personas consumidoras y usuarias ${ }^{3}$. Tras un primer capítulo, llamado "disposiciones generales"

\footnotetext{
*Sección redactada bajo la dirección de José Ignacio MORILLO-VELARDE PÉREZ.
}

${ }^{1}$ Realizado por $\mathrm{M}^{\mathrm{a}}$ del Carmen NÚÑEZ LOZANO (Universidad de Huelva).

${ }^{2}$ El carácter básico de la totalidad del Real Decreto resulta, a nuestro juicio, discutible, así como su densidad normativa habida cuenta tal carácter básico. No obstante, el Decreto que vamos a comentar le reconoce este carácter en su Exposición de Motivos.

${ }^{3}$ El Decreto hace uso del denominado lenguaje no sexista y se aparta del criterio asumido por la Ley 13/2003, en cuya Exposición de Motivos se advierte que "se ha optado por la utilización del término consumidor para referirse tanto a los consumidores propiamente dichos como a los usuarios, ya que, aunque quizás se pudiera ensayar una distinción teórica entre ambas nociones, el régimen jurídico de protección que se les otorga es idéntico y su reiterada utilización a lo largo del texto haría su lectura más difícil sin aportar matices diferenciadores importantes. Únicamente se mantiene la referencia a consumidores y usuarios en la denominación de determinados órganos y en lo referente a las organizaciones o asociaciones de consumidores y usuarios". 
-en el que se precisa el objeto y ámbito de aplicación y se efectúa una remisión a las definiciones que de producto seguro, producto inseguro, riesgo, productor y distribuidor contiene el art. 2 del RD 1801/2003- se aborda en el capítulo segundo lo concerniente a la Red de Alerta; el capítulo tercero disciplina las medidas preventivas y también las advertencias y requerimientos; contiene el Decreto además dos disposiciones adicionales y dos disposiciones finales.

En la exposición que sigue, para una mejor comprensión de las cuestiones que plantea el Decreto, analizaremos en primer lugar las medidas preventivas y las advertencias y requerimientos para, a continuación, ocuparnos de la Red de Alerta. Antes, sin embargo, precisaremos el ámbito de aplicación del Decreto y efectuaremos unas consideraciones previas de índole conceptual.

II.- El Decreto se aplica a todo producto que se suministre o se ponga a disposición de las personas consumidoras y usuarias, de forma gratuita o en el marco de una actividad comercial o de una prestación de servicios (art. 1.2). Se excluyen los productos que dispongan de una regulación específica con el mismo objeto y, expresamente, los alimentos, los medicamentos y los productos sanitarios, así como los productos usados que se suministren como antigüedades o para ser reparados o reacondicionados antes de su utilización, siempre que el proveedor informe de ello claramente a la persona a la que suministre el producto (art. 1.3 y 4 ).

Como antes se avanzó, el Decreto hace suyas las definiciones de producto seguro, producto inseguro, riesgo, productor y distribuidor que contiene el art. 2 del RD 1801/2003. Sin embargo, los primeros términos no se utilizan en la disposición, que adopta como referencia el de "riesgo inaceptable", que es el que utiliza la Ley 13/2003, cuya aprobación precedió en unos días a la del RD 1801/2003.

La Exposición de Motivos de la Ley, tras destacar que "el punto central de la regulación" del "derecho a la protección de la salud y seguridad" es "la prohibición de que los bienes o servicios destinados a los consumidores representen un riesgo inaceptable para su salud y seguridad", advierte que "sin duda alguna, la noción de riesgo inaceptable es un complejo concepto jurídico indeterminado, que conlleva un cierto margen de apreciación por parte de los poderes públicos" y aclara que "se ha optado por esa denominación que es la empleada por la Unión Europea". Sin embargo, en la Directiva 2001/95/CE no se contiene la expresión "riesgo inaceptable" y sí los términos 
"producto seguro", "producto peligroso" y "riesgo grave", cuyos significados recoge el RD 1801/2001, con alguna matización en lo que concierne al "riesgo grave", que se define en el contexto más general del concepto de riesgo, y al uso de la expresión "producto peligroso", que se cambia por la de "producto inseguro".

"Producto seguro" es, a tenor del art. 2.a) del RD 1801/2003, "cualquier producto que, en condiciones de utilización normales o razonablemente previsibles, incluidas las condiciones de duración y, si procede, de puesta en servicio, instalación y de mantenimiento, no presente riesgo alguno o únicamente riesgos mínimos compatibles con el uso del producto y considerados admisibles dentro del respeto de un nivel elevado de protección de la salud y de la seguridad de las personas, habida cuenta, en particular, de los siguientes elementos: $1^{\circ}$ ) Las características del producto, entre ellas su composición y envase; $2^{\circ}$ ) El efecto sobre otros productos, cuando razonablemente se pueda prever la utilización del primero junto con los segundos; $3^{\circ}$ ) La información que acompaña al producto. En particular, el etiquetado, los posibles avisos e instrucciones de uso y eliminación; las instrucciones de montaje y, si procede, instalación y mantenimiento, así como cualquier otra indicación o información relativa al producto; $4^{\circ}$ ) La presentación y publicidad del producto; $5^{\circ}$ ) Las categorías de consumidores que estén en condiciones de riesgo en la utilización del producto, en particular los niños y las personas mayores"; se aclara que "la posibilidad de alcanzar niveles superiores de seguridad o de obtener otros productos que presenten menor grado de riesgo no será razón suficiente para considerar que un producto es inseguro". "Producto inseguro" es, según el art. 2.b) del RD, "cualquiera que no responda a la definición de producto seguro". Y sobre el riesgo señala el art. 2.c) que es la "posibilidad de que los consumidores y usuarios sufran un daño para su salud o seguridad, derivado de la utilización, consumo o presencia de un producto. Para calificar un riesgo desde el punto de vista de su gravedad, entre otras posibles circunstancias, se valorará conjuntamente la probabilidad de que se produzca un daño y la severidad de éste. Se considerará riesgo grave aquel que en virtud de tales criterios exija una intervención rápida de las Administraciones públicas, aun en el caso de que los posibles daños para la salud y seguridad no se materialicen inmediatamente" 4 .

\footnotetext{
${ }^{4}$ El art. 3 del RD 1801/2003 fija los parámetros de referencia para la evaluación de la seguridad de los productos, estableciendo determinadas presunciones sobre cuando un producto es seguro y cuando es inseguro. Vid. también el art. 60 de la Ley 13/2003, sobre la presunción de riesgo inaceptable para la salud o seguridad, que da entrada además a la cualidad de peligrosidad del producto.
} 
En nuestra opinión, el término "riesgo inaceptable" resulta muy acertado y oportuno por su expresividad. En nuestra sociedad, en la que no existe el riesgo cero, resulta la diáfana la contraposición entre riesgos que son inaceptables y riesgos que son aceptables. $\mathrm{Y}$ aunque es cierto que no se define el riesgo inaceptable ni en la Ley 13/2003 ni en este Decreto, facilitan sin duda la integración del concepto las definiciones que de producto seguro, producto inseguro, riesgo y riesgo grave proporciona el RD 1801/2003. Cuestión distinta es, como habremos de ver, que el Decreto haya acertado plenamente en la descripción de los presupuestos de hecho de las intervenciones administrativas que regula, en particular por lo que concierne a la Red de Alerta.

III.-Las medidas administrativas preventivas proceden ante situaciones de riesgo inaceptable para la seguridad de las personas consumidoras y usuarias, con la finalidad de eliminar o reducir, en su caso, dicho riesgo (art. 10.1, primer párrafo $)^{5}$. El enunciado del presupuesto de hecho coincide con el del art. 59.2 de la Ley 13/2003, aunque no con el del art. 10 del RD 1801/2003, que se refiere al incumplimiento de los deberes generales regulados en el RD, si bien a la postre vincula cada medida a la inseguridad del producto, cierta o sobre la que pesen indicios razonables.

A nuestro juicio, resulta más idónea la descripción del presupuesto de hecho de la normativa andaluza ${ }^{6}$, en la medida en que el $\mathrm{RD}$ recoge múltiples deberes (arts. 4 a 6) sin especificar cuáles son los generales, de modo que aquélla ofrece mayor seguridad jurídica mediante la utilización del concepto referido que, aunque indeterminado, es fácilmente determinable, teniendo en cuenta el principio de cautela ${ }^{7}$ y mediante la integración de las nociones

\footnotetext{
${ }^{5}$ Aunque el Decreto no contiene referencias a ello, conviene precisar que las medidas no tienen carácter sancionador ni se vinculan a la apertura de un procedimiento de este tipo. Ello es un indudable acierto de la legislación de protección de los consumidores y usuarios. Merece la pena destacar los arts. 58.3 y 4 de la Ley 13/2003 y 8.4 del Real Decreto 1801/2003.

${ }^{6}$ Que se acomoda también a lo previsto en la Directiva 2001/95/CE, cuyo art. 8 hace pivotar la adopción de medidas de este tipo de la peligrosidad eventual o cierta de los productos, lo que se reconduce a la noción de "riesgo inaceptable.

${ }^{7}$ Que no se menciona en el Decreto; sí lo recoge el art. 8.2, segundo párrafo, del Real Decreto. Vid. M. REBOLLO PUIG y M. IZQUIERDO CARRASCO, "El principio de precaución y la defensa de los consumidores", Documentación Administrativa núms. 265 y 266, 2003, pp. 211 ss, en las que destacan que la ausencia de consagración expresa del principio en la legislación de protección de consumidores y usuarios no impide su aplicación.
} 
de producto seguro, producto inseguro y riesgo grave, a las que no en balde se remite el art. 2 del Decreto, como ya hubo ocasión de precisar. Lo anterior se corrobora si se repara en que una de las situaciones que contempla el Real Decreto, la de "producto sobre el que existan indicios razonables de que pueda ser inseguro" [art. 10.a)], no tiene por qué implicar el incumplimiento de un deber general si a la postre los indicios no se confirman; esa misma situación, sin embargo, se integraría sin dificultad en la noción de "riesgo inaceptable" del Decreto andaluz, máxime en la medida en que el art. 58.1 de la Ley 13/2003 precisa que la adopción de medidas procede "cuando existan claros indicios de riesgo".

Las medidas, cuya adopción resulta obligada si concurre el presupuesto de hecho, según se desprende claramente del empleo del verbo "deber" en el primer párrafo del art. 10.1, pueden consistir en: a) Establecer condiciones previas a la comercialización que favorezcan la seguridad del producto; b) Suspender o prohibir la oferta, promoción o venta; c) Inmovilizar cautelarmente o retirar los productos ofertados a las personas consumidoras y usuarias $\mathrm{y}$, si fuese necesario, acordar su destrucción en condiciones adecuadas; d) Clausurar establecimientos; e) Establecer las medidas que garanticen la plena eficacia de las anteriores (art. 10.1, segundo párrafo). Se trata de un elenco que, al igual que el del art. 10 del RD 1801/2003, tiene carácter ejemplificativo, sin perjuicio de que por su complitud resulte difícil imaginar otras medidas posibles; ha de notarse que el Decreto andaluz incluye la clausura de establecimientos y que el Real Decreto describe con mayor detalle las medidas.

Rigen, evidentemente, los principios generales de la actividad limitativa de policía, aunque el Decreto se cuida de especificar, haciendo suyo el contenido del art. 8.2 del Real Decreto, que "las medidas adoptadas, así como los medios para su ejecución o efectividad, deberán ser congruentes con los motivos que las originan, proporcionadas con los riesgos que afronten y, de entre las que reúnan esos requisitos, se adoptarán las que resulten menos restrictivas de la libertad de circulación de mercancías y prestación de servicios, de la libertad de empresa y demás derechos afectados" (art. 10.2).

Los órganos competentes en la materia son: 1) En el ámbito de la Administración de la Junta de Andalucía, las personas titulares de las Delegaciones Provinciales de la Consejería competente en materia de consumo. Corresponde a la persona titular de la Dirección General competente en materia de consumo la coordinación de las actuaciones de las Delegaciones Provinciales, pudiendo ordenar lo necesario para la extensión 
de las medidas, determinar los trámites a llevar a cabo por éstas y, en su caso, avocar la competencia, a efectos de garantizar que las decisiones adoptadas tengan eficacia en todo el territorio de la Comunidad (art. 11.1); 2) En el ámbito municipal, los órganos municipales competentes según lo previsto en el art. 67.2 de la Ley $13 / 2003^{8}$ (art. 11.2); 3) Tienen competencia para la adopción de estas medidas administrativas las personas que tengan encomendadas las tareas de inspección en materia de consumo, de conformidad con el art. 46.c) $)^{9}$ y $68.2^{10}$ de la Ley $13 / 2003$ (art. 11).

El procedimiento para la adopción de las medidas se regula en los arts. 13 a 15 , que de suyo no regulan un procedimiento completo sino que prescriben que se siga "el correspondiente procedimiento tramitado conforme a lo dispuesto en la Ley 30/1992" (art. 14.1), de modo que sólo incorporan determinadas precisiones, algunas ciertamente interesantes desde la perspectiva de la economía del procedimiento y de la racionalidad de la actuación administrativa, pero en absoluto originales, puesto que el Decreto se limita a efectuar, con las excepciones que luego detallaremos, una refundición e incluso síntesis de lo prescrito en la Ley 13/2003 y en el RD 1801/2003. Antes de ocuparnos de ello, conviene destacar que cabe la adopción de las medidas provisionales que sean imprescindibles para garantizar la efectiva protección de la seguridad de las personas consumidoras y usuarias, con carácter previo a la iniciación del procedimiento, en el acuerdo de inicio o durante su instrucción (art. 13.2, que expresamente invoca el art. 68 de la Ley 13/2003 ${ }^{11}$ ).

\footnotetext{
${ }^{8}$ A tenor del mismo, "los órganos municipales, sin menoscabo de lo establecido en el apartado anterior [que prevé la competencia de los órganos de defensa del consumidor de la Administración autonómica], podrán adoptar estas medidas cuando la situación a la que respondan sea estrictamente de ámbito local y se pueda afrontar en su totalidad dentro del término municipal. En caso contrario, sólo podrán adoptar provisionalmente las medidas circunscritas a su ámbito territorial que sean urgentes, poniéndolo en conocimiento inmediato de los órganos autonómicos para que tomen las decisiones procedentes. También colaborarán en la aplicación de las que se tomen en un ámbito superior".

${ }^{9}$ Que habilita a los inspectores de consumo para adoptar provisionalmente, en caso de urgencia, medidas adecuadas a las irregularidades que hayan detectado.

${ }^{10}$ Que específicamente contempla la adopción de las medidas, con carácter provisional y antes de la iniciación del procedimiento, incluso por los servicios de inspección.

${ }^{11} \mathrm{El}$ art. 68 regula con cierto detalle las medidas provisionales, de modo que en este art. 13.2 del Decreto es especialmente visible la labor de mero resumen. El precepto es también más escueto que el art. 11.2, 3 y 4 del Real Decreto, de modo que omite importantes garantías que, naturalmente, habrán de ser observadas en cualquier caso.
} 
El procedimiento se inicia de oficio, por propia iniciativa o como consecuencia de orden superior, a petición razonada de otros órganos o por denuncia (art. 13.1). Si el acto administrativo afecta a sujetos determinados, se les dará audiencia y las demás posibilidades de intervención que corresponden a personas interesadas según la Ley 30/1992 (art. 14.2, que transcribe el art. 64.2 de la Ley 13/2003). No será necesaria la realización de los trámites de audiencia y prueba cuando hayan sido cumplimentados ante el mismo supuesto de hecho y ante idéntica medida en el procedimiento administrativo que haya seguido otra Administración autonómica, la Administración del Estado u otra de ámbito superior (art. 14.3, que trae causa del art. $11.1 \mathrm{del}$ Real Decreto ${ }^{12}$ ). En este caso, en el expediente se incluirá una identificación del procedimiento y órgano donde se realizaron dichos trámites, así como toda la documentación que se haya recibido al respecto (art. 14.3, que trae causa también del art. 11.1 del Real Decreto). Tampoco se realizará el trámite de audiencia en aquellos casos en que fuera imposible la localización o identificación de las personas productoras o distribuidoras del producto o prestadoras del servicio de que se trate, causantes del riesgo (art. 14.3, que recoge lo obvio y que se inspira en el art. 64.1 de la Ley 13/2003, aunque de suyo no lo sigue, porque lo que establece el precepto legal, menos obvio y más acertado en nuestra opinión, es que no se ha de seguir procedimiento administrativo "en aquellos casos en que fuera imposible la localización o identificación de los responsables de la producción, distribución o comercialización de bienes y servicios y a cualquier otro responsable del mantenimiento o existencia del riesgo").

El procedimiento se tramitará y resolverá con la celeridad que la adopción de este tipo de medidas requieren, y finalizará mediante resolución del órgano en cada caso competente, sin perjuicio del plazo máximo para resolver y notificar la resolución expresa establecido en el artículo 64.2 de la Ley 13/2003 (art. 15.1 ${ }^{13}$ ). La resolución, en el ámbito de la Administración de la

\footnotetext{
${ }^{12} \mathrm{Si}$ bien completa su contenido mediante la referencia a "la Administración del Estado u otra de ámbito superior". Con ello se amplía la previsión del art. 11.1 a los casos recogidos en el art. 14.1 del mismo Real Decreto, de modo que, de suyo, lo que debe tener lugar en estos casos es la ejecución autonómica de la medida ya adoptada por el Estado, en su caso previa decisión de la Comisión. No estaríamos, por tanto, ante un procedimiento para adoptar una medida, sino ante el procedimiento de ejecución de la medida ya adoptada por otra instancia.

${ }^{13}$ La llamada a la celeridad responde a los arts. 8.1del Real Decreto y 58.1 de la Ley. El plazo del art. 64.2 es de seis meses.
} 
Junta de Andalucía, no agota la vía administrativa, por lo que cabe recurso de alzada; respecto de los municipios, con respeto a su autonomía, se indica que cabrá el recurso que corresponda (art. 15.2).

El Decreto precisa que en cualquier momento en que los afectados decidan voluntariamente cumplir su deber de adoptar las medidas adecuadas para evitar los riesgos en la forma que el órgano administrativo competente considere que queda garantizada la seguridad de las personas consumidoras y usuarias, se podrá dar por finalizado el procedimiento y dictar resolución en la que así se haga constar (art. 14.2, que reproduce el art. 11.5 del Real Decreto, con omisión de la referencia que hace éste a la salud de las personas $\left.^{14}\right)$.

IV.- En sede de medidas administrativas preventivas, el Decreto regula las advertencias y requerimientos, en desarrollo del art. 66.1 de la Ley 13/2003 y mediante la transcripción del art. 9.2 y 3 del Real Decreto ${ }^{15}$.

Proceden cuando se observe un incumplimiento de leyes o reglamentos que afecten a los intereses de las personas consumidoras y usuarias pero que no genere riesgos inaceptables. En este supuesto, la Administración puede advertir al transgresor de la situación ilegal y de su obligación de cesar en su conducta, y requerirle para que subsane los defectos detectados (art. 12.1).

El requerimiento debe incluir el resultado a que debe llegarse, el plazo para alcanzarlo y el seguimiento que se realizará o la forma en que los produc-

\footnotetext{
${ }^{14}$ Puesto que las medidas de que tratamos son las previstas en el art. 59.2 de la Ley 13/2003, que se refiere a situaciones de riesgo inaceptables para la seguridad. A las que lo son para la salud se refiere el art. 59.1 de la Ley, que sólo prevé que los órganos de defensa de los consumidores puedan adoptar medidas cautelares, con ulterior comunicación a las autoridades sanitarias, que son las competentes. Ha de advertirse, no obstante, de la artificiosa distinción en estos ámbitos entre salud y seguridad (como han subrayado M. REBOLLO RUIG y M. IZQUIERDO CARRASCO, ob. cit. pp. 192 ss.); a esta realidad responde la disposición adicional segunda del Decreto, que presupone actuaciones de los órganos de consumo respecto de productos que presenten un riesgo inaceptable para la salud de las personas consumidoras y usuarias.

${ }^{15} \mathrm{El}$ art. 12.1 del Decreto viene a coincidir con el art. 66.1 de la Ley. Los apartados 2 y 3 del citado art. 12 son transcripción prácticamente literal de los apartados 2 y 3 del art. 9 del Real Decreto. Como nota curiosa, cabe destacar que el Real Decreto se refiere a productores y distribuidores, pues no tensiona el lenguaje con la perspectiva de género; el Decreto es fiel en su transcripción, de modo que menciona también a los productores y distribuidores, lo que podría suscitar la cuestión de si el precepto no es de aplicación a las productoras y distribuidoras.
} 
tores o distribuidores deberán justificar ante los órganos administrativos competentes las actuaciones que, en su caso, emprendan, dejando a éstos la posibilidad de elegir la forma y los medios para lograr el resultado en tanto que ello no esté predeterminado legal o reglamentariamente, y sin perjuicio de que dichos órganos puedan recomendar la forma en que entiendan que puede ser subsanado el incumplimiento (art. 12.2). Si el productor o el distribuidor no actúa en el plazo establecido, o si su actuación no es satisfactoria, o si resulta insuficiente, se podrán acordar medidas administrativas preventivas (art. 12.3).

En nuestra opinión, aunque tanto la Ley como el Decreto utilizan el verbo "poder", ha de interpretarse que la Administración, conocedora de la situación de incumplimiento, debe dirigir la advertencia y el requerimiento en todo caso, con la finalidad de que cese la misma y, por tanto, la afección a los intereses de las personas consumidoras y usuarias. De este modo, serán mayores las probabilidades, lógicamente, de que no se lleguen a producir riesgos inaceptables. Ha de considerarse que si en última instancia la mejor protección es una eficaz prevención, la Administración debe actuar con diligencia y, por esta vía, atajar desde el primer momento los incumplimientos, antes de que se provoque cualquier situación de riesgo inaceptable y aún por la mera probabilidad de que se pueda generar ${ }^{16}$.

V.- La Red de Alerta de Andalucía se configura como un instrumento de colaboración, cooperación y coordinación entre los distintos órganos de la Administración de la Junta de Andalucía con competencias en materia de consumo; y entre éstos y las Entidades Locales (art. 3.1). Su objeto es facilitar la comunicación e intercambio rápido de información entre los órganos de las Administraciones indicadas y entre éstas y el organismo de la Administración General del Estado competente en materia de consumo, sobre actuaciones que se adopten en relación con los productos de consumo que puedan suponer un riesgo para la salud y la seguridad de las personas consumidoras y usuarias (arts. 1.1 y 4 ).

Se basa en un sistema de intercambio rápido de información, que el Decreto caracteriza como en forma de red $^{17}$, cuyos nudos o puntos de

\footnotetext{
${ }^{16}$ Por ello no nos parece del todo correcto el art. 40.2 de la Ley 13/2003, que vincula la intervención administrativa en los casos de incumplimiento de la normativa a la susceptibilidad de lesionar o poner en peligro de cualquier forma los derechos de los consumidores.

17 Aunque, de suyo, no es exactamente la imagen de red la que conviene a la estructura y funcionamiento del sistema, como a continuación se verá.
} 
contacto son las unidades administrativas de las Administraciones Públicas de Andalucía que participan, desde las que se transmiten y reciben las comunicaciones respecto de las actuaciones que se adopten en relación con los productos de consumo referidos (art. 3.2).

La coordinación y organización de la Red corresponde a la Dirección General competente en materia de consumo ${ }^{18}$, que se designa como punto de contacto autonómico del Sistema de Intercambio Rápido de Información $(\operatorname{SIRI})^{19}$. Es la encargada de evaluar la información generada por el punto de contacto de ámbito provincial y de decidir si la información recibida se comunica a la Administración General del Estado a través del Sistema. También le corresponde transmitir a todos los puntos de contacto de la Red la información recibida de esta Administración a través del SIRI (art. 5.1).

El punto de contacto de ámbito provincial es el Servicio competente en materia de inspección de consumo de cada una de las Delegaciones provinciales de la Consejería competente en materia de consumo. Le corresponde la

\footnotetext{
${ }^{18}$ Anuncia la disposición adicional primera que la Dirección General competente en materia de consumo aprobará directrices o guías de funcionamiento de la Red de Alerta de Andalucía, donde se determinen, entre otros aspectos, los medios técnicos -especialmente con carácter telemático- que deban emplearse para la transmisión de las comunicaciones, el formulario tipo, la información que éste debe contener, los procedimientos de aplicación, los plazos de notificación y comunicación de actuaciones y los criterios para clasificar y ordenar las comunicaciones según su objeto y urgencia. No nos resistimos a manifestar nuestra inquietud en cuanto a la necesidad de clasificar y ordenar las comunicaciones según su urgencia, pues acaso se quiera indicar con ello que en nuestra Comunidad Autónoma proliferan las situaciones de riesgo inaceptable de modo tal que hay que atenderlas según la urgencia de cada una, desde la premisa, por demás, que todas ellas deben ser urgentes (como se desprende de los presupuestos para la utilización de la Red y del contenido de las comunicaciones, aspectos que más adelante trataremos); nos tranquiliza, no obstante, la constatación de que la disposición adicional cuarta del Real Decreto 1801/2003 tiene un contenido análogo, de modo que acaso esta disposición adicional primera sea más fruto del mimetismo que de la existencia cotidiana en Andalucía de situaciones de riesgos inaceptables.

${ }^{19}$ El SIRI lo crea el art. 19 del RD 1801/2003, con la finalidad de facilitar la comunicación y el intercambio de información de las actuaciones que se adopten en caso de riesgo grave. El citado artículo impone a los órganos autonómicos la obligación de realizar las comunicaciones que contempla, que se trasladan a los demás órganos competentes y, en su caso, a la Comisión a través del sistema RAPEX. A través del SIRI se da traslado también de las comunicaciones procedentes de la Comisión. Cada Comunidad Autónoma debe comunicar al Instituto Nacional de Consumo el punto de contacto único para todos los productos incluidos en el ámbito de aplicación del Real Decreto que se designe a tal efecto, así como los mecanismos que establezca para garantizar su disponibilidad permanente.
} 
coordinación, evaluación y traslado de las notificaciones derivadas de su propio funcionamiento y del de los puntos de contacto de las Entidades Locales integradas en la Red de Alerta de su provincia al punto de contacto autonómico (art. 5.2).

Por lo que respecta al nivel local, pueden integrarse en la Red a través de puntos de contacto todas las Entidades Locales que de conformidad con lo previsto en el art. 97.1.f) y g) de la Ley 13/2003 comuniquen a la Consejería competente en materia de consumo la asunción de las competencias relativas a la Inspección de Consumo y a la adopción de medidas administrativas preventivas para garantizar los derechos e intereses de las personas consumidoras y usuarias $^{20}$. Para la integración, han de comunicar a la citada Dirección General su punto de contacto que, como sabemos, será coordinado por la Delegación Provincial de la Consejería con competencias en materia de consumo (art. 5.3).

Corresponde a cada Administración Pública el establecimiento de los mecanismos necesarios para garantizar una disponibilidad permanente de los puntos de contacto y de las autoridades competentes para la adopción y confirmación, modificación o levantamiento de las medidas administrativas preventivas que tengan como finalidad la protección de la salud y seguridad de las personas consumidoras y usuarias (art. 5.4).

A través de la Red se comunican las actuaciones previstas en el art. 19 del RD 1801/2003 ante situaciones de riesgo inaceptables para la salud y la seguridad de las personas consumidoras y usuarias y en relación con los productos de consumo, que son las siguientes: a) Toda medida administrativa preventiva que se haya adoptado; b) Los requerimientos que se hayan dirigido a productores o distribuidores ${ }^{21}$, siempre que incluyan una recomendación de la forma en que el órgano administrativo competente entiende que debe ser subsanado el incumplimiento que ha generado el riesgo grave; c) Las actuaciones voluntarias que hayan emprendido los productores y distribuidores en virtud de los deberes impuestos por el Decreto (art. 6).

${ }^{20}$ El art. 97.5 de la Ley 13/2003 señala que las Corporaciones Locales, respecto de las competencias que puedan asumir conforme a lo dispuesto en la Ley, comunicarán a la Consejería competente en materia de protección de los consumidores su aceptación por acuerdo plenario.

${ }^{21}$ Nótese de nuevo la disfuncionalidad que plantea el uso del lenguaje no sexista cuando se transcriben literalmente disposiciones provenientes de otras normativas, ahora el art. 19.2 del Real Decreto. 
Para realizar la comunicación es preciso disponer de datos suficientes que permitan apreciar unos indicios razonables de la existencia de riesgo grave. La comunicación ha de ser lo más completa posible y contener, como mínimo, los siguientes datos: a) El motivo de la comunicación; b) La información que permita identificar el producto que ha generado la situación de riesgo, c) Una descripción del riesgo y los resultados de toda prueba, análisis o ensayo que se hayan realizado, así como sus conclusiones, de modo que permitan su evaluación; d) La identificación de las personas productoras o distribuidoras del producto o prestadoras del servicio de que se trate; e) Toda información relevante que, en su caso, se haya podido obtener de las personas referidas; f) La información que se posea sobre las cadenas de comercialización y distribución del producto o servicio, g) Según lo que proceda, el carácter y la duración de las medidas administrativas preventivas adoptadas, de las actuaciones voluntarias emprendidas por las personas productoras, distribuidoras o prestadoras de servicios, o bien el contenido de la advertencia o requerimiento previo formulado a aquéllas (art. 7.1).

Finalmente, la información de riesgos a los consumidores se produce en los términos que contempla el art. 8, que desarrolla el art. 70 de la Ley $13 / 2003$ y que dispone que, sin perjuicio de la utilización de cualesquiera otros medios que se consideren necesarios, la Dirección General, tras la verificación que regula el art. 7.2, publicará la información básica sobre todo producto objeto de comunicación en la Red de Alerta en la correspondiente página web ubicada en la sede electrónica de la Administración de la Junta de Andalucía (como sabemos, www.juntadeandalucia.es).

Varios comentarios merece esta regulación de la Red de Alerta.

En primer lugar, hay que destacar que su creación no la impone el Real Decreto ni la Ley. El primero crea y regula el SIRI, pero no impone la existencia de un sistema análogo en cada Comunidad Autónoma. La segunda prevé lógicamente la comunicación entre órganos y Administraciones Públicas (art. 63), pero no predetermina que haya de ser a través de un sistema como el que regula, a semejanza del SIRI, el Decreto. Desde esta perspectiva, ha de valorarse positivamente la iniciativa andaluza.

Ello no obsta a que se deban puntualizar algunas imperfecciones de la regulación.

Una de ellas, en nuestra opinión, es la utilización de la palabra "red" para designar el sistema de alerta, pues de suyo éste adopta propiamente una estructura vertical, no reticular, al igual que el SIRI. Ello se confirma en el art. 
7.2 del Decreto, que prescribe que las Delegaciones Provinciales, antes de remitir a la Dirección General toda comunicación que le haya sido dirigida por una Entidad Local para su inclusión en la Red de Alerta, verificarán que dicha comunicación cumple con lo dispuesto en los arts. 6 y 7.1, recabándose, en su caso, las aclaraciones, subsanaciones o informaciones adicionales necesarias. Las mismas facultades corresponden a la Dirección General respecto de las comunicaciones recibidas de las Delegaciones Provinciales. De ahí que no resulte correcto el art. 4 al precisar que el objeto de la red es facilitar la comunicación e intercambio de información entre los distintos órganos que menciona el art. 3, pues de suyo la comunicación es vertical y bidireccional, nunca multilateral. Ello no obstante, cada Administración Pública comunicante es responsable de toda la información proporcionada (art. 7.3).

Mayores reparos ofrece otro aspecto de la normativa, que es la concerniente a la determinación de qué ha de comunicarse y cuándo hay que comunicar. El Decreto presenta aquí dificultades de interpretación al poner en conexión lo previsto para la Red de Alerta con la regulación de las medidas administrativas preventivas y de las advertencias y requerimientos, aparentemente clara y que por ello se ha expuesto en primer término.

El Decreto, en efecto, al ocuparse de la creación y configuración de la Red, así como de su objeto, precisa que a través de ella se comunican actuaciones adoptadas en relación con productos que puedan suponer un riesgo para la salud y la seguridad (arts. 3.2 y 4). Sin embargo, al tratar de las actuaciones que son objeto de la comunicación condiciona ésta a la existencia de situaciones de riesgo inaceptables (art. 6). Y a continuación, cuando detalla el contenido de la comunicación, cambia el presupuesto de hecho mediante la referencia a la disposición de los datos suficientes que permitan apreciar unos indicios suficientes de la existencia del riesgo grave (art. 7.1).

Por otra parte, lo que se comunican son las medidas administrativas preventivas adoptadas, los requerimientos y las actuaciones voluntarias (art. 6): las primeras proceden ante situaciones de riesgo inaceptable; las segundas ante incumplimientos que no generan riesgo inaceptable; mientras que las terceras no están ni siquiera bien delimitadas en el Decreto, pues tanto pueden referirse a actuaciones en ejecución de los convenios de colaboración que prevé el art. 9 en el mismo capítulo que regula la Red de Alerta ${ }^{22}$, como

\footnotetext{
${ }^{22}$ A tenor del citado art. 9, "la Administración de la Junta de Andalucía podrá celebrar convenios de colaboración con otras Administraciones Públicas, con organizaciones empresariales y otras entidades, en aras de garantizar la seguridad de los productos puestos a disposición de las personas consumidoras y usuarias".
} 
a las medidas adoptadas en el curso del procedimiento de adopción de medidas administrativas preventivas.

Se plantea así qué sentido tiene condicionar la comunicación de medidas administrativas preventivas a la disposición de indicios razonables de la existencia del riesgo grave (art. 7), si precisamente éstas se adoptan ante situaciones de riesgo inaceptable (art. 10) ${ }^{23}$. O cómo se conjuga la supeditación de la comunicación de requerimientos cursados ante incumplimientos que no generan situaciones de riesgo inaceptable (art. 12), a la existencia de situaciones de riesgo inaceptables (art. 6) ${ }^{24}$ o de indicios razonables de la existencia de riesgo grave (art. 7). Y también se puede cuestionar si existe una equivalencia entre la situación de riesgo inaceptable o riesgo grave y la eventualidad de que un producto suponga un riesgo, que es la hipótesis que describen los arts. 3.2 y $4^{25}$.

${ }^{23}$ Una posible explicación, que creemos que ha descartarse, es la de que para la activación de la Red de Alerta no rija el principio de cautela y sí, en cambio, para la adopción de las medidas. En nuestra opinión, el principio de cautela rige también para la activación de la Red.

${ }^{24}$ Por cierto que no serían supuestos idénticos los de "situaciones de riesgo inaceptable" (art. 10 por ejemplo) y "situaciones de riesgos inaceptables” (art. 6). Se podrían plantear distintas hipótesis interpretativas: 1) situaciones de riesgos inaceptables son situaciones de riesgo, sea cual sea el calificativo de éste, desde la premisa de que cualquier situación de riesgo es en sí misma inaceptable ("inaceptables" sería un epíteto); 2) son situaciones en las que los riesgos son inaceptables, por contraposición a situaciones, también de riesgo, pero en las que el riesgo es aceptable ("inaceptables sería un calificativo"); 3) se trata de una errata, de modo que se quiso decir "situaciones de riesgo inaceptable", lo que vendría avalado por la circunstancia de que el plural "inaceptables" se utiliza sólo en este art. 6.

25 Estos problemas no se plantean con el RD, puesto que los presupuestos de hecho de las medidas administrativas de reacción (preventivas en la terminología del Decreto) y de las advertencias y requerimientos son diferentes entre sí (incumplimiento de los deberes generales, cifrados en la inseguridad del producto e incumplimiento del resto de deberes, respectivamente) y compatibles todos ellos, nominalmente al menos, con el presupuesto que determina la utilización de la red de alerta estatal o SIRI, que es el de riesgo grave. 